\title{
Outcome of incidentally detected airway nodules
}

\author{
Hyung-Jun Kim ${ }^{1,2}$, Deog Kyeom Kim³,3, Young Whan Kim ${ }^{1,4}$, Yeon Joo Lee ${ }^{1,2}$, \\ Jong Sun Park ${ }^{1,2}$, Young-Jae Cho ${ }^{1,2}$, Se Joong Kim ${ }^{1,2}$, Ho Il Yoon ${ }^{1,2}$, \\ Jae Ho Lee ${ }^{1,2}$ and Choon-Taek Lee ${ }^{1,2}$
}

\begin{abstract}
Affiliations: ${ }^{1}$ Dept of Internal Medicine, Seoul National University College of Medicine, Seoul, Republic of Korea. ${ }^{2}$ Division of Pulmonary and Critical Care Medicine, Dept of Internal Medicine, Seoul National University Bundang Hospital, Seongnam-Si, Republic of Korea. ${ }^{3}$ Division of Pulmonary and Critical Care Medicine, Dept of Internal Medicine, Seoul Metropolitan Government-Seoul National University Boramae Medical Center, Seoul, Republic of Korea. 'Division of Pulmonary and Critical Care Medicine, Dept of Internal Medicine, Seoul National University Hospital, Seoul, Republic of Korea.
\end{abstract}

Correspondence: Choon-Taek Lee, 82 Gumi-ro 173 Beon-gil, Bundang-gu, Seongnam-Si, Gyeonggi-do 13620, Republic of Korea. E-mail: ctleedsnu.ac.kr

ABSTRACT Low-dose chest computed tomography (LDCT) screening increased detection of airway nodules. Most nodules appear to be secretions, but pathological lesions may show similar findings. The National Comprehensive Cancer Network (NCCN) recommends repeating LDCT after 1 month and proceeding to bronchoscopy if the nodules persist. However, no reports exist about incidentally detected airway nodules. We investigated the significance of airway nodules detected by LDCT screening.

We screened patients with incidental airway nodules detected by LDCT in the Seoul National University Hospital group. The characteristics of computed tomography, bronchoscopy, pathology and clinical findings were analysed.

Among 53036 individuals who underwent LDCT screening, 313 (0.6\%) had airway nodules. Of these, 186 (59.4\%) were followed-up with chest computed tomography and/or bronchoscopy. Seven (3.8\%) cases had significant lesions, including leiomyoma $(n=2)$, endobronchial tuberculosis $(n=2)$, chronic inflammation $(n=1)$, hamartoma $(n=1)$ and benign granuloma $(n=1)$. The remaining 179 lesions were transient, suggesting that they were secretions.

The use of LDCT for lung cancer screening demonstrated the low incidence of airway lesions. Most lesions were transient secretions. True pathological lesions were rare, and no malignant lesion was found. The current recommendation of the NCCN guideline is a reasonable approach that can avoid unnecessary bronchoscopy.

@ERSpublications

The current NCCN guidelines for incidentally found airway nodules seem to be a reasonable approach http://ow.ly/Ywn6A

This article has supplementary material available from erj.ersjournals.com

Received: Nov 272015 | Accepted after revision: Feb 132016 | First published online: March 312016

Conflict of interest: None declared.

Copyright OERS 2016 


\section{Introduction}

Nodules of the tracheobronchial tree are produced by various diseases, including malignancy, benign tumour, infection, trauma, other idiopathic aetiologies such as sarcoidosis, and transient insignificant lesions such as secretions [1-4]. Tracheobronchial tumours are rare, representing $0.4 \%$ of all body tumours [5], and $10 \%$ are reported to be benign [6]. Primary tumours of the trachea represent $0.1 \%$ of body tumours [7]. Early recognition of the airway lesion is crucial for timely intervention to minimise complications related to airway compromise [1]. Furthermore, airway metastasis from extrapulmonary tumours may be the first manifestation of the neoplasm in $\sim 5 \%$ of cases, as shown in a recent study [8]. Diverse types of malignancies may present as airway invasion or metastasis $[9,10]$.

Early detection of an airway lesion is difficult because these lesions are usually invisible on simple chest radiographs, and symptoms are not evident in the early stage of the disease. Therefore, computed tomography (CT) is recommended to facilitate early intervention when airway lesions are suspected [1]. Several CT findings have been suggested to indicate the nature of airway nodules. Mucus materials present as nodules of low attenuation and have a bubbly appearance, usually in the dependent portion of the airway, while malignant tumours present as polypoid nodules with eccentric wall thickening and benign tumours present as popcorn calcifications with internal fat material $[5,6]$.

Despite the recent clinical implementation of low-dose chest CT (LDCT) for lung cancer screening in high-risk patients $[11,12]$, guidelines for cancer screening of airway lesions are not clearly established. The current National Comprehensive Cancer Network (NCCN) guidelines suggest that if endobronchial nodules are suspected on LDCT, follow-up LDCT should be performed after 1 month following vigorous coughing, and bronchoscopic examination is recommended if the lesions persist [13]. However, no evidence has been presented to support the recommendation of a follow-up CT. Previous studies have shown that a CT scan can help to distinguish endobronchial lung cancer in $79-83 \%$ of cases $[14,15]$. However, these reports were retrospective reviews of diagnosed cancer patients using contrast-enhanced CT. No previous studies are available on the clinical impact of LDCT on airway tumours.

The aims of this study were to evaluate the clinical significance of incidentally detected airway nodules from LDCT screening for lung cancer in health promotion centres, to determine the role of follow-up CT, to provide evidence to support the current NCCN guidelines, and to assess whether LDCT is an effective method for detecting early bronchogenic cancer in the airway.

\section{Material and methods \\ Study design and participants}

We reviewed patients who received LDCT for the purpose of lung cancer screening at Seoul National University Bundang Hospital (Seongnam-Si, Republic of Korea), Seoul National University Hospital and Seoul National University Boramae Medical Center (Seoul, Republic of Korea) (the Seoul National University Hospital group) between May 2003 and February 2015; January 1999 and February 2015; and January 2007 and February 2015. This duration was determined by the oldest picture archiving and communicating system data available in each hospital.

This study was conducted in accordance with the amended Declaration of Helsinki and was reviewed by the institutional review board in each hospital (the protocol numbers are B-1501/282-110, H-1505-089-673 and 20150706/16-2015/86-081). Informed consent was not obtained, but patient records and information were anonymised and de-identified prior to analysis. Among LDCT interpretation, keywords implying airway nodules, such as endobronchial, endotracheal, luminal, bronchoscopy, mucus and airway, etc., were initially screened. All screened LDCTs were reviewed to confirm the cases of real airway nodules. Airway nodules were defined as a roughly spherical opacity confined within the lumen of the airway (bronchus and trachea) [16]. Luminal narrowing, atelectasis without airway nodules and nodules within bronchiectatic airways were excluded.

\section{Measurements and definition}

Demographic and clinical characteristics were collected, including smoking status, follow-up method and interval, initial and final diagnosis and referral to a pulmonologist. Characteristics of the nodules on CT, such as the longest diameter, Hounsfield unit, shape and margin were recorded. LDCTs were performed according to the standardised protocols of each medical centre (1-5 mm thickness, with radiation dose of 1-2 mSv). Length was measured as the longest diameter using the preset lung window setting of each medical centre (level -500--700 HU; width 1500-2000 HU). The Hounsfield unit was measured by drawing a round region of interest in the preset mediastinal window setting of each centre (level 25-45 HU; width 250-400 HU) while avoiding grossly visible heterogeneous lesions, such as air, fat and calcification. The location of the nodule was classified as anterior or posterior based on its position relative 
to an imaginary line crossing the midline of the airway lumen [16]. The initial diagnosis of the nodule was based on the radiologist's interpretation and classified as a secretion, true lesion or both possible.

Final diagnosis was established by CT follow-up and/or bronchoscopy, with or without pathologic confirmation. Secretion was confirmed by the disappearance or alteration of a nodule on a follow-up CT. Finally, the airway nodules were classified as either true lesions or secretions. The clinical and radiological characteristics of each group were compared.

\section{Analysis}

All statistical analysis was performed using SPSS 22.0 (IBM, Armonk, NY, USA). The variables were analysed using the Pearson Chi-squared or Fisher's exact tests for categorical variables and the t-test or Mann-Whitney U-test for continuous variables.

\section{Results}

\section{Baseline and clinical characteristics}

53036 individuals underwent LDCT in three medical centres. Characteristics such as smoking history, previous cancer history, sex and age were unknown. Initially, 5880 patients were selected by searching keywords of radiologists' interpretations. After excluding false-positive selections by individual CT review, $313(0.6 \%)$ patients were found with airway nodules, and of these patients, $186(59.4 \%)$ were followed-up for proper diagnosis (fig. 1). The reasons for loss of follow-up were high suspicion of secretion (81.9\%), failure of the patient to return for follow-up (10.2\%), CT study too recent for follow-up to be conducted (3.9\%) and referral to other hospitals (3.1\%) (table 1).

Of the 186 patients followed-up, $165(88.7 \%)$ were male, with an average age of 56.1 years. $63 \%$ of the patients were current smokers, $14.5 \%$ were former smokers and $19.9 \%$ were never-smokers. No subjective symptoms were found in the majority of the patients $(86.0 \%)$. Sputum was found in $6.5 \%$ of patients, followed by cough $(2.7 \%)$, dyspnoea $(2.2 \%)$, chest soreness $(1.1 \%)$, throat pain $(0.5 \%)$ and palpitation (0.5\%) (table 2).

\section{CT findings of airway nodules}

The average length of the nodules was $8.48 \mathrm{~mm}$ and the median Hounsfield units were -36.5 . The trachea was the most common location (49.5\%), followed by the left main bronchus (13.4\%) and the right lower lobar bronchus (11.8\%). $54 \%$ of the nodules were found posteriorly within the airways, and the presence of a circumscribed margin (55.9\%) was slightly more common than an uncircumscribed margin (44.1\%). An obtuse angle (61.3\%) and an oval shape (51.1\%) were the most common. The initial CT interpretation

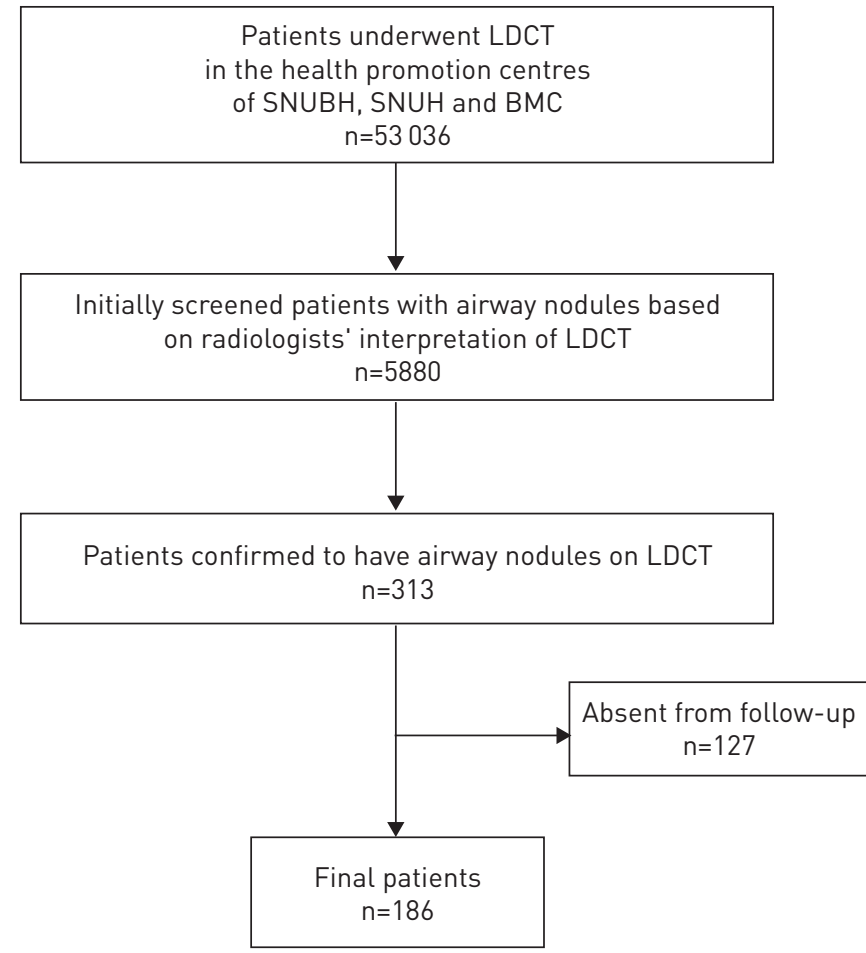

FIGURE 1 Flowchart of the patient selection process. SNUBH: Seoul National University Bundang Hospital; SNUH: Seoul National University Hospital; BMC: Boramae Medical Center; LDCT: low-dose chest computed tomography. 


\section{TABLE 1 Reasons for loss to follow-up}

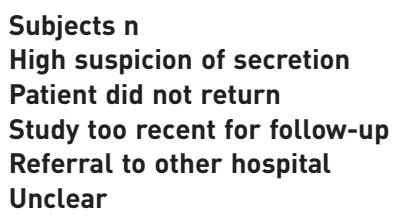

Data are presented as $\mathrm{n}(\%)$, unless otherwise stated.

was secretion in $69.9 \%$ of cases and indeterminate nodule in $26.9 \%$; true lesions were strongly suggested only in $3.2 \%$ of cases (table 3 ).

\section{Diagnosis}

Follow-up of endobronchial or endotracheal nodules was conducted in 186 patients using CT (86.6\%), bronchoscopy (9.7\%) or both (3.8\%) (fig. 2). The median follow-up interval was 9 months, and $31.7 \%$ of the patients were referred to a pulmonologist for further evaluation. Finally, 179 (96.3\%) of the 186 patients were confirmed to have nodules composed of secretory materials. The remaining seven true airway nodules were found to be leiomyoma $(n=2)$, endobronchial tuberculosis $(n=2)$, chronic inflammation $(n=1)$, hamartoma $(n=1)$ and benign granuloma $(n=1)$ (table 4). No malignant nodules were found.

The characteristics of the seven patients with true airway nodules are summarised in table 5. Four patients had a history of smoking, and two had clinical symptoms including sputum and cough. Six cases were initially suspected to have true airway lesion by the responding radiologist. For example, case 2 had an airway nodule located between the carina and the left main bronchus, and the follow-up CT showed a persistent lesion, leading to a bronchoscopic evaluation. It also showed a polypoid mass and biopsy results confirmed that the lesion was leiomyoma (online supplementary fig. S1a). In contrast, the CT findings in case 6 were initially suspected to be caused by secretion; however, follow-up CT showed a persistent lesion and bronchoscopic evaluation led to the diagnosis of a hamartoma (online supplementary fig. S1b). A case of endobronchial tuberculosis (case 4) was confirmed by sputum smear and culture for Mycobacterium tuberculosis, while the other (case 5) was diagnosed by coexisting parenchymal tuberculosis and size reduction of the nodule after anti-tuberculosis medication.

Comparison between secretion and a true airway lesion

We compared the clinical and radiological characteristics between secretions and true airway lesions. True lesions tended to have a higher density $(\mathrm{HU})$ than secreted material $(\mathrm{p}=0.004)$, and the initial radiologists opinion was significantly correlated with the final diagnosis $(\mathrm{p}=0.001)$. A history of smoking, an obtuse angle and an ovoid shape suggested secretion rather than a true lesion, but these findings were not statistically significant due to an insufficient number of cases (table 6).

\section{TABLE 2 Baseline and clinical characteristics}

$\begin{array}{lc}\text { Male } & 165(88.7) \\ \text { Age years } & 56.10 \pm 10.63 \\ \text { Smoking } & \\ \text { Current smoker } & 118(63.4) \\ \text { Former smoker } & 27(14.5) \\ \text { Never-smoker } & 37(19.9) \\ \text { Unknown } & 4(2.2) \\ \text { Duration of smoking pack-years } & 32.60 \pm 17.45 \\ \text { Symptoms } & \\ \text { None } & 160(86.0) \\ \text { Sputum } & 12(6.5) \\ \text { Cough } & 5(2.7) \\ \text { Dyspnoea } & 4(2.2) \\ \text { Chest soreness } & 2(1.1) \\ \text { Throat pain } & 1(0.5) \\ \text { Palpitation } & 1(0.5)\end{array}$

Data are presented as $\mathrm{n}(\%)$ or mean \pm SD. ${ }^{\#}$ : multiple symptoms possible. 


\section{TABLE 3 Characteristics of airway nodules on computed tomography}

\section{Size $\mathrm{mm}$}

Density HU

Location

Central airway

Peripheral airway

Position

Posterior

Anterior

Unclear

Shape

Oval

Lobulated

Round

Complex

Initial diagnosis

Secretion

Secretion or true lesion

True lesion
$8.48 \pm 3.73$

$-36.5(-106.25-5.25)$

134 (72.0)

52 (28.0)

$101(54.3)$

65 (34.9)

20 (10.8)

95 (51.1)

35 (18.8)

34 (18.3)

22 (11.8)

130 (69.9)

50 (26.9)

6 (3.2)

Data are presented as mean $\pm \mathrm{SD}$, median (interquartile range) or $\mathrm{n}(\%) .{ }^{\#}$ : includes the trachea, right main bronchus and left main bronchus.

\section{Discussion}

Lung cancer screening using LDCT has been proven to be effective for detecting early-stage lung cancer in high-risk individuals, although some uncertainty exists regarding the potential harms of screening and the generalisability of the results $[11,17,18]$. LDCT was an effective screening method for detecting early lung cancer presenting as peripheral nodules (solitary pulmonary nodules, including ground-glass opacity nodules). However, most clinicians and researchers doubt that LDCT will be useful for detecting early lung cancer arising in the airways (trachea and bronchus).

Several studies have investigated lesions of the tracheobronchial tree. Lesions with a round or lobulating shape, an uncircumscribed margin, or a CT value of $\geqslant 21.7 \mathrm{HU}$ may indicate true lesions [16]. In other studies, CT findings of bronchial carcinoid tumours have been described [19], and differentiation of anthracofibrosis from endobronchial tuberculosis using CT has proven to be viable [20]. Furthermore, increased fluorodeoxyglucose positron emission tomography/CT uptake at the obstruction site indicates a high probability of malignancy, with certain types of cancer showing a significantly higher standard uptake value than others [21, 22]. However, these studies were based on contrast-enhanced CT with standard-dose radiation, or nuclear imaging with radioactive materials.

Our study is the first to analyse the incidence of incidentally detected airway nodules using LDCT. 53036 patients underwent LDCT for purpose of lung cancer screening at three medical centres over a $\sim 10$-year period. $313(0.6 \%)$ of these cases were found to have endobronchial or endotracheal nodules. This result is similar to previous reports regarding the general incidence of endobronchial tumours $[5,7]$.

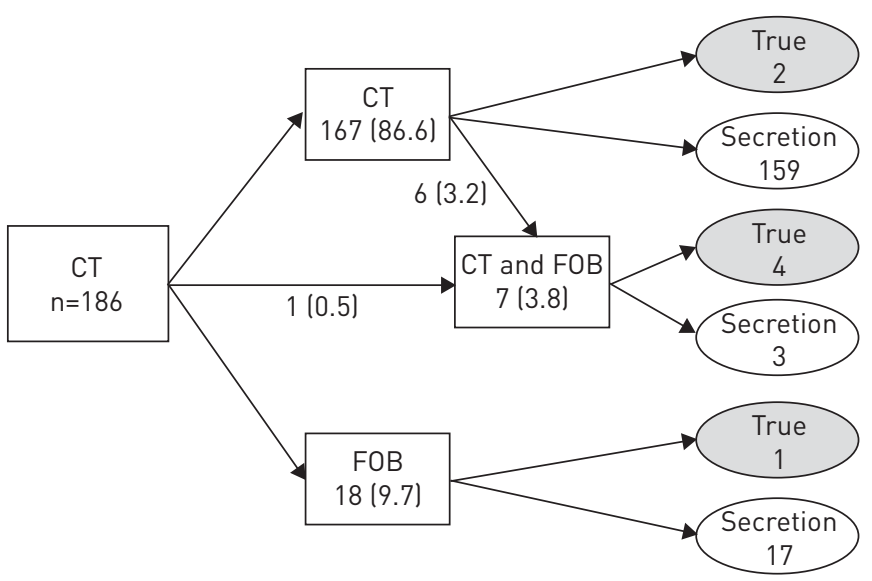

FIGURE 2 Follow-up method of incidentally found airway nodules. Data are presented as n (\%). CT: computed tomography; FOB: fibre optic bronchoscopy. 
TABLE 4 Findings of follow-up

$\begin{array}{lc}\text { Follow-up method } & \\ \text { CT } & 161(86.6) \\ \text { Bronchoscopy } & 18(9.7) \\ \text { Both } & 7(3.8) \\ \text { Follow-up interval months } & 9(3-14) \\ \text { Bronchoscopic evaluation } & 25(13.4) \\ \text { Referral to pulmonologist } & 59(31.7) \\ \text { Final diagnosis } & 179(96.3) \\ \text { Secretion } & 2(1.1) \\ \text { Leiomyoma } & 2(1.1) \\ \text { Endobronchial tuberculosis } & 1(0.5) \\ \text { Chronic inflammation } & 1(0.5) \\ \text { Hamartoma } & 1(0.5) \\ \text { Benign granuloma } & \end{array}$

Data are presented as $\mathrm{n}(\%)$ or median (interquartile range). CT: computed tomography.

Bronchoscopy is the procedure of choice for the evaluation of airway tumours [23]. Flexible fibre optic bronchoscopy is a relatively safe procedure, but it is still invasive and expensive. It may induce procedureor anaesthesia-related complications such as hypoxaemia $(0.2-21 \%)$, arrhythmia (1-10\%), post-biopsy bleeding (0.12-7.5\%), pneumothorax or pneumomediastinum (1-6\%), fever (0.9-2.5\%) and death $(0.1-0.2 \%)$ [24].

CT has a role in defining and treating lung cancer with endoluminal invasion [25], and it may be used instead in selected cases when the patient has a contraindication to bronchoscopy or has refused the procedure [26]. A previous study attempting to determine whether CT scans could be substituted for bronchoscopy failed to detect endobronchial tumours in $17 \%$ of patients [14]. CT has the disadvantage of a lower sensitivity for detecting endobronchial nodules, but substituting CT for bronchoscopy can have benefits in terms of reducing the related complications.

Our study has shown that incidentally detected airway nodules were mostly secretions (96.3\%), and true lesions were not found to be malignant. Considering this incidence and the potential adverse effects of the bronchoscopic procedure, CT follow-up would be sufficient in most cases and bronchoscopy should be undertaken selectively. Of the 186 patients, 161 (86.6\%) underwent follow-up CT initially, and 18 (9.7\%) of them underwent bronchoscopy primarily as a follow-up method. The other seven (3.8\%) patients underwent bronchoscopy after CT follow-up due to persistent nodules. Of the total 25 bronchoscopic exams, only five $(2.9 \%)$ cases were diagnosed with significant airway lesions, and $20(11.7 \%)$ were found to be secretions. The more prudent application of bronchoscopy did not cause a significant delay in diagnosis. These data could provide a rationale for the current NCCN guideline: repeat LDCT after 1 month with vigorous coughing when endobronchial nodules are suspected on screening CT and further bronchoscopy should be considered if the nodule is not resolved [13].

\begin{tabular}{|c|c|c|c|c|c|c|c|c|c|}
\hline Case & Sex & $\begin{array}{l}\text { Age } \\
\text { years }\end{array}$ & Smoking & Symptoms & $\begin{array}{c}\text { Initial } \\
\text { interpretation }\end{array}$ & Follow-up & FOB findings & Pathology & $\begin{array}{c}\text { Final } \\
\text { diagnosis }\end{array}$ \\
\hline 1 & M & 75 & Former & None & Both $^{\#}$ & Both" & $\begin{array}{c}\text { Elevation of normal } \\
\text { mucosa }\end{array}$ & $\begin{array}{l}\text { Chronic active } \\
\text { inflammation }\end{array}$ & $\begin{array}{c}\text { Chronic } \\
\text { inflammation }\end{array}$ \\
\hline 2 & $M$ & 41 & Current & None & True lesion & Both & Polypoid mass & Leiomyoma & Leiomyoma \\
\hline 5 & M & 46 & Never & Cough & True lesion & CT & Not done & Not done & EBTB \\
\hline 6 & M & 52 & Former & None & Secretion & Both" & Polypoid mass & $\begin{array}{c}\text { Mature cartilaginous } \\
\text { tissue }\end{array}$ & Hamartoma \\
\hline 7 & $\mathrm{~F}$ & 64 & Never & None & Both ${ }^{\#}$ & Both & Nodular lesion & $\begin{array}{l}\text { Negative for } \\
\text { malignant cells }\end{array}$ & $\begin{array}{c}\text { Benign } \\
\text { granuloma }\end{array}$ \\
\hline
\end{tabular}

Data are presented as $n$, unless otherwise stated. FOB: fibre optic bronchoscopy; M: male; F: female; CT: computed tomography; EBTB: endobronchial tuberculosis. " : both secretion and true lesions possible; " 


\section{TABLE 6 Comparison between secretions and true airway lesions}

\begin{tabular}{|c|c|c|c|}
\hline & Secretion & True lesion & p-value \\
\hline Subjects & 179 & 7 & \\
\hline Male & $160(89.4)$ & $5(71.4)$ & 0.180 \\
\hline Age years & $56.31 \pm 10.47$ & $50.71 \pm 14.07$ & 0.337 \\
\hline Ever smoking & $141(80.6)$ & $4(57.1)$ & 0.150 \\
\hline Size mm & $8.53 \pm 3.77$ & $7.09 \pm 2.41$ & 0.172 \\
\hline Density (HU) & $-39(-110--1.70)$ & $30(-9.33-44)$ & 0.004 \\
\hline Location & & & 0.097 \\
\hline Central airway & $131(73.2)$ & $3(42.9)$ & \\
\hline Peripheral airway & $48(26.8)$ & $4(57.1)$ & \\
\hline Angle & & & 0.104 \\
\hline Obtuse & 112 (64.0) & $2(28.6)$ & \\
\hline Not obtuse & $63(36.0)$ & $5(71.4)$ & \\
\hline Shape & & & 0.063 \\
\hline Ovoid & 93 (52.0) & $1(14.3)$ & \\
\hline Acute or unclear & 86 (48.0) & 6 (85.7) & \\
\hline Initial diagnosis & & & 0.001 \\
\hline Secretion & $136(76.0)$ & 1 (14.3) & \\
\hline Unsure & $43(24.0)$ & $6(85.7)$ & \\
\hline Symptoms & 22 (12.3) & $2(28.6)$ & 0.224 \\
\hline
\end{tabular}

Data are presented as $\mathrm{n}, \mathrm{n}(\%)$, mean $\pm \mathrm{SD}$ or median (interquartile range), unless otherwise stated.

\#: includes the trachea, right main bronchus and left main bronchus.

Although only a small number of cases were confirmed as true airway lesions, a higher HU value may indicate true lesions rather than the presence of secreted materials, and this is in accordance with a previous report [16]. Furthermore, except for one case of a hamartoma that was initially diagnosed as a secretion, six other cases were initially suspected to have true lesions by the attending radiologist. As the data were obtained from three different medical centres, we can assume that the interpretations of qualified radiologists correspond with the final diagnosis of incidentally detected airway nodules.

Interestingly, we did not find any cases of early bronchogenic cancer presenting as airway nodules. We propose several possibilities that may explain the absence of malignancy.

First, the low incidence of airway malignancies may be due to the characteristics of our study population, which are similar to the general population. Despite recent recommendations suggesting LDCT in high-risk populations, the criteria for LDCT are not well established in South Korea. The decision to perform LDCT mostly depends on the patient's personal needs regardless of risk factors for lung cancer such as a history of smoking. However, when high-risk smokers were chosen as a subgroup, only 47 cases of airway nodules were found, and all turned out to be secretory materials [11].

Second, LDCT is not a suitable method for detecting early bronchogenic cancer in contrast to peripheral lung nodules. Among the patients who were screened and diagnosed with lung cancer in the National Lung Screening Trial, only $\sim 1.8 \%$ of them were found to have atelectasis upon LDCT [27]. Neither endobronchial nor endotracheal nodules were mentioned. In the NELSON study of 7155 participants, 34 patients had interval cancers, roughly defined as lung cancers diagnosed after false negative LDCT results. Among the 34, five cancers were not detected initially due to their location in the endobronchial lumen [28]. Endobronchial nodules are still considered as blind spots on screening CTs [29].

This study has some limitations. This is a retrospective study, and the screened population is not a high-risk group for lung cancer. $40 \%$ of the patients with airway nodules were not properly followed-up for diagnosis.

In summary, the use of LDCT for lung cancer screening demonstrated the low incidence of airway nodules $(0.6 \%)$. Secretions were predominant, and true lesions were extremely rare. Furthermore, none of the true lesions were confirmed as malignancies. Most lesions could be diagnosed via CT follow-up only, and primary bronchoscopic evaluation was not helpful. Therefore, the current recommendation of LDCT follow-up according to the NCCN guideline for endobronchial nodules appears to be a reasonable approach that can prevent unnecessary bronchoscopy.

\section{Acknowledgements}

We appreciate the members of the Dept of Internal Medicine, Seoul National University College of Medicine (Seoul, Republic of Korea) for sharing their ideas and comments. 


\section{References}

1 Marom EM, Goodman PC, McAdams HP. Focal abnormalities of the trachea and main bronchi. AJR Am J Roentgenol 2001; 176: 707-711.

2 Upadya A, Tilluckdharry L, Nagy CD, et al. Endobronchial pseudo-tumour caused by herpes simplex. Eur Respir J 2005; 25: 1117-1120.

3 De Falco Alfano D, Totaro M, Zagà C, et al. Endobronchial lipomatous hamartoma diagnosed on computed tomography scan in young new mother - a case report. Int J Surg Case Rep 2014; 5: 1113-1116.

4 Wilson RW, Kirejczyk W. Pathological and radiological correlation of endobronchial neoplasms: part I, benign tumors. Ann Diagn Pathol 1997; 1: 31-46.

5 Park CM, Goo JM, Lee HJ, et al. Tumors in the tracheobronchial tree: CT and FDG PET features. Radiographics 2009; 29: 55-71.

6 El-Kersh K, Perez RL, Gauhar U. A 63-year-old man with a chronic cough and an endobronchial lesion. Diagnosis: endobronchial hamartoma. Chest 2014; 145: 919-922.

$7 \quad$ Macchiarini P. Primary tracheal tumours. Lancet Oncol 2006; 7: 83-91.

8 Marchioni A, Lasagni A, Busca A, et al. Endobronchial metastasis: an epidemiologic and clinicopathologic study of 174 consecutive cases. Lung Cancer 2014; 84: 222-228.

9 Lee SH, Jung JY, Kim do H, et al. Endobronchial metastases from extrathoracic malignancy. Yonsei Med J 2013; 54: 403-409.

10 Kurishima K, Kagohashi K, Miyazaki K, et al. Small cell lung cancer with endobronchial growth: a case report. Oncol Lett 2013; 6: 553-555.

11 Bach PB, Mirkin JN, Oliver TK, et al. Benefits and harms of CT screening for lung cancer: a systematic review. JAMA 2012; 307: 2418-2429.

12 Moyer VA. Screening for lung cancer: U.S. Preventive Services Task Force recommendation statement. Ann Intern Med 2014; 160: 330-338.

13 Network NCC. Lung Cancer Screening (Version 1.2016). www.nccn.org/professionals/physician_gls/pdf/lung screening.pdf Date last accessed: August 19, 2015.

14 Aristizabal JF, Young KR, Nath H. Can chest CT decrease the use of preoperative bronchoscopy in the evaluation of suspected bronchogenic carcinoma? Chest 1998; 113: 1244-1249.

15 Han NJ, Song KS, Lee KH, et al. Superficial endobronchial lung cancer: radiologic-pathologic correlation. Korean J Radiol 2002; 3: 229-234.

16 Hong SR, Lee YJ, Hong YJ, et al. Differentiation between mucus secretion and endoluminal tumors in the airway: analysis and comparison of CT findings. AJR Am J Roentgenol 2014; 202: 982-988.

17 Aberle DR, Adams AM, Berg CD, et al. Reduced lung-cancer mortality with low-dose computed tomographic screening. N Engl J Med 2011; 365: 395-409.

18 Mazzone P. Lung cancer screening: examining the issues. Cleve Clin J Med 2012; 79: Suppl. 1, eS1-eS6.

19 Jeung MY, Gasser B, Gangi A, et al. Bronchial carcinoid tumors of the thorax: spectrum of radiologic findings. Radiographics 2002; 22: 351-365.

20 Park HJ, Park SH, Im SA, et al. CT differentiation of anthracofibrosis from endobronchial tuberculosis. AJR Am J Roentgenol 2008; 191: 247-251.

21 Cho A, Hur J, Kang WJ, et al. Usefulness of FDG PET/CT in determining benign from malignant endobronchial obstruction. Eur Radiol 2011; 21: 1077-1087.

22 Aquino SL, Halpern EF, Kuester LB, et al. FDG-PET and CT features of non-small cell lung cancer based on tumor type. Int J Mol Med 2007; 19: 495-499.

23 Rabahi MF, Ferreira AA, Reciputti BP, et al. Fiberoptic bronchoscopy findings in patients diagnosed with lung cancer. J Bras Pneumol 2012; 38: 445-451.

24 Geraci G, Pisello F, Sciumè C, et al. Le complicanze della fibrobroncoscopia. Revisione della letteratura. [Complication of flexible fiberoptic bronchoscopy. Literature review]. Ann Ital Chir 2007; 78: 183-192.

25 Hicks RJ, Lau E, Alam NZ, et al. Imaging in the diagnosis and treatment of non-small cell lung cancer. Respirology 2007; 12: 165-172.

26 Naidich DP, Lee JJ, Garay SM, et al. Comparison of CT and fiberoptic bronchoscopy in the evaluation of bronchial disease. AJR Am J Roentgenol 1987; 148: 1-7.

27 Aberle DR, DeMello S, Berg CD, et al. Results of the two incidence screenings in the National Lung Screening Trial. N Engl Med 2013; 369: 920-931.

28 Horeweg N, Scholten ET, de Jong PA, et al. Detection of lung cancer through low-dose CT screening (NELSON): a prespecified analysis of screening test performance and interval cancers. Lancet Oncol 2014; 15: 1342-1350.

29 Scholten ET, Horeweg N, de Koning HJ, et al. Computed tomographic characteristics of interval and post screen carcinomas in lung cancer screening. Eur Radiol 2015; 25: 81-88. 